

A. N. Abyzov, V. T. Le, C. Q. Truong, A. A. Tuganbaev, Modules coinvariant under the idempotent endomorphisms of their covers, Sibirsk. Mat. Zh., 2019, Volume 60, Number 6, 1191-1208

DOI: https://doi.org/10.33048/smzh.2019.60.601

Use of the all-Russian mathematical portal Math-Net.Ru implies that you have read and agreed to these terms of use http://www . mathnet.ru/eng/agreement

Download details:

IP: 52.23 .180 .231

April 26, 2023, 15:31:17

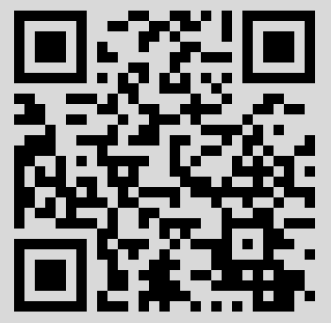


Сибирский математический журнал

Ноябрь-декабрь, 2019. Том 60, № 6

УДК 512.553

\title{
МОДУЛИ, КОИНВАРИАНТНЫЕ ОТНОСИТЕЛЬНО ИДЕМПОТЕНТНЫХ ЭНДОМОРФИЗМОВ СВОИХ НАКРЫТИЙ
}

\author{
А. Н. Абызов, В. Т. Ле, \\ К. К. Чюонг, А. А. Туганбаев
}

\begin{abstract}
Аннотация. Изучаются модули, коинвариантные относительно идемпотентных эндоморфизмов своих накрытий. Вводятся и изучаются обобщения дискретных и непрерывных модулей с помощью теории накрытий и оболочек модулей. В качестве приложения рассмотрены случаи плоских накрытий, инъективных оболочек и чисто инъективных оболочек.
\end{abstract}

DOI 10.33048/smzh.2019.60.601

Ключевые слова: квазиинъективный модуль, квазипроективный модуль, дискретный модуль, непрерывный модуль, автоморфизм-коинвариантный модуль, оболочка, накрытие.

\section{1. Введение}

Многие важные классы модулей, близких к инъективным (соответственно проективным), могут быть определены с помощью инъективных оболочек (соответственно проективных накрытий). Квазиинъективные модули введены в [1] как модули, инвариантные относительно эндоморфизмов своих инъективных оболочек. В этой же работе показано, что модуль $M$ квазиинъективен в точности тогда, когда каждый гомоморфизм из подмодуля модуля $M$ в модуль $M$ продолжается до некоторого эндоморфизма $M$. Модуль, инвариантный относительно автоморфизмов своей инъективной оболочки, называется автоморфизм-инвариантным. Впервые автоморфизм-инвариантные модули над конечномерными алгебрами были изучены Диксоном и Фуллером в [2]. В [3] введено понятие псевдоинъективного модуля, т. е. модуля, в котором каждый мономорфизм из подмодуля модуля $M$ в модуль $M$ продолжается до некоторого эндоморфизма $M$. В [4] показано, что модуль $M$ является псевдоинъективным в точности тогда, когда $M$ - автоморфизм-инвариантный модуль. Двойственное понятие к автоморфизм-инвариантным модулям под названием

Исследование А. Н. Абызова выполнено за счет средств субсидии, выделенной Казанскому (Приволжскому) федеральному университету для выполнения государственного задания в сфере научной деятельности, проект № 1.13556.2019/13.1. Le Van Thuyet and Truong Cong Quynh were supported by the Vietnam National Foundation for Science and Technology Development (NAFOSTED) under grant number 101.04-2018.02 and would like to thank Hue university for the received support. Truong Cong Quynh was the Funds for Science and Technology Development of the University of Danang under project number B2017-DN03-08. Исследование А. А. Туганбаева выполнено за счет гранта Российского научного фонда (проект 16-11-10013).

(c) 2019 Абызов А. Н., Ле В. Т., Чюонг К. К., Туганбаев А. А. 
автоморфизм-коинвариантные (или дуально автоморфизм-инвариантные) модули недавно изучено в [5-7].

Непрерывные модули и их расширения - квазинепрерывные модули - введены и изучены в [8-11] как модульные аналоги непрерывных и квазинепрерывных колец, рассмотренных Утуми в [12]. В [13] показано, что модуль $M$ квазинепрерывен в точности тогда, когда модуль $M$ инвариантен относительно идемпотентных эндоморфизмов своей инъективной оболочки. Многие важные свойства непрерывных, квазинепрерывных модулей и их дуальных аналогов отражены в [14-19].

Общая теория модулей, инвариантных и коинвариантных относительно автоморфизмов соответственно своей оболочки и своего накрытия, была в последнее время развита в [20-22]. Теория модулей, инвариантных относительно идемпотентных эндморфизмов своей оболочки, изучена в [23].

В разд. 2 исследуются модули, коинвариантные относительно идемпотентных эндоморфизмов своих накрытий. Для произвольного класса $\mathscr{X}$ правых $R$-модулей, замкнутого относительно изоморфных образов, вводится и изучается понятие $\mathscr{X}$-модуля со свойством подъема. В случае, когда $R-$ совершенное справа кольцо и $\mathscr{X}$ - класс проективных правых $R$-модулей, класс $\mathscr{X}$ модулей со свойством подъема совпадает с классом правых $R$-модулей со свойством подъема. В разд. 3 показано, что дискретные (соответственно непрерывные) модули могут быть определены с помощью инъективных (соответственно проективных) оболочек (соответственно накрытий). Этот факт позволяет дать определения понятий $\mathscr{X}$-непрерывного модуля и $\mathscr{X}$-дискретного модуля, которые являются естественными и широкими обобщениями понятий дискретного модуля и непрерывного модуля. Изучены общие свойства колец эндоморфизмов $\mathscr{X}$-непрерывных и $\mathscr{X}$-дискретных модулей. Показано, что каждый $\mathscr{X}$ непрерывный ( $\mathscr{X}$-дискретный) модуль конечно заменяем. В качестве приложения рассмотрены случаи проективных накрытий, инъективных оболочек и чисто инъективных оболочек.

Радикал Джекобсона кольца $R$ обозначается через $J(R)$. Тот факт, что $N$ является подмодулем (соответственно малым подмодулем, существенным подмодулем) модуля $M$ будем обозначать через $N \leq M$ (соответственно через $\left.N \ll M, N \leq_{e} M\right)$. Радикал Джекобсона правого $R$-модуля $M$ обозначается через $J(M)$.

В работе используются стандартные понятия и факты теории колец и модулей (см., например, [18, 24-26]).

\section{2. $\mathscr{X}$-идемпотентно коинвариантные модули}

Будем предполагать, что $\mathscr{X}$ - некоторый класс правых $R$-модулей, замкнутый относительно изоморфных образов и прямых слагаемых. Гомоморфизм $g: X \rightarrow M$ правых $R$-модулей называется $\mathscr{X}$-накрытием модуля $M$, если

(1) $X \in \mathscr{X}$ и для всякого гомоморфизма $g^{\prime}: X^{\prime} \rightarrow M$, где $X^{\prime} \in \mathscr{X}$, существует гомоморфизм $h: X^{\prime} \rightarrow X$, для которого выполнено равенство $g^{\prime}=g h$;

(2) для каждого эндоморфизма $h: M \rightarrow M$ из равенства $g=g h$ следует, что $h$ - автоморфизм.

Модуль $M$ называется модулем со свойством подгема, если для каждого подмодуля $N$ модуля $M$ существуют такие подмодули $M_{1}, M_{2}$ модуля $M$, что выполнены условия

$$
M=M_{1} \oplus M_{2}, \quad M_{1} \leq N, \quad M_{2} \cap N \ll M_{2} .
$$


Модуль $M$ называется $D 3$-модулем, если $X \cap Y$ - прямое слагаемое в $M$ для любых таких прямых слагаемых $X$ и $Y$ в $M$, что $X+Y=M$. Модуль $M$ называется квазидискретным, если он является одновременно модулем со свойством подъема и $D 3$-модулем.

Предложение 1 [16, предложение 4.45]. Пусть $u: P \rightarrow M-$ проективное накрытие модуля $M$. Для модуля $M$ следующие утверждения эквивалентны:

(1) $M-$ квазидискретный модуль;

(2) $M$ - идемпотент-коинвариантный модуль, т. е. $\alpha(\operatorname{Ker}(u)) \subseteq \operatorname{Ker}(u)$ для каждого идемпотентного эндоморфизма $\alpha$ модуля $P$.

Пусть $M$ - правый $R$-модуль. Модуль $M$ называется $\mathscr{X}$-идемпотентно коинвариантным, если существует такое $\mathscr{X}$-накрытие $u: X \rightarrow M$, что для каждого идемпотента $g \in \operatorname{End}(X)$ существует эндоморфизм $f: M \rightarrow M$, для которого коммутативна диаграмма

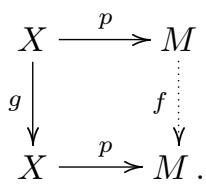

Лемма 2. Пусть $p: X \rightarrow M$ - эпиморфное $\mathscr{X}$-накрытие правого $R$-модуля $M$. Если $M-\mathscr{X}$-идемпотентно коинвариантный модуль, то для каждого идемпотента $g^{2}=g \in \operatorname{End}(X)$ существует такой однозначно определенный гомоморфизм $f \in \operatorname{End}(M)$, что $f p=p g$ и $f^{2}=f$.

ДокАЗАтЕльство. Существуют гомоморфизмы $f, f^{\prime} \in \operatorname{End}(M)$, для которых выполнены равенства $f p=p g$ и $f^{\prime} p=p(1-g)$. Тогда $f^{\prime} f p=f^{\prime} p g=0$. Поскольку $p$ - эпиморфизм, то $f^{\prime} f=0$. Так как $p=p g+p(1-g)=f^{\prime} p+f p=$ $\left(f^{\prime}+f\right) p$, то id $=f^{\prime}+f$. Таким образом, $f=f^{2} \in \operatorname{End}(M)$. При этом поскольку $p$ - эпиморфизм, гомоморфизм $f$ однозначно определен.

Лемма 3. Пусть $p: X \rightarrow M-$ эпиморфное $\mathscr{X}$-накрытие модуля $M$. Следующие условия равносильны:

(1) $M-\mathscr{X}$-идемпотентно коинвариантный модуль;

(2) $g(\operatorname{Ker}(p)) \leq \operatorname{Ker}(p)$ для каждого идемпотентного эндоморфизма модуля $X$.

ДокАЗАТЕЛЬСТво. Импликация $(1) \Rightarrow(2)$ очевидна.

$(2) \Rightarrow(1)$ Предположим, что $g=g^{2} \in \operatorname{End}(X)$. Тогда $g(\operatorname{Ker}(p)) \leq \operatorname{Ker}(p)$. Рассмотрим гомоморфизм $\psi: X / g(\operatorname{Ker}(p)) \rightarrow M$, определенный согласно правилу: $\psi(x+g(\operatorname{Ker}(p))=p(x)$ для всех $x \in X$. Поскольку $p$ - эпиморфизм, для каждого $m \in M$ существует такой элемент $x \in X$, что $m=p(x)$. Рассмотрим следующее отображение:

$$
\phi: M \rightarrow X / g(\operatorname{Ker}(p)), \quad m \mapsto g(x)+g(\operatorname{Ker}(p)) .
$$

Легко видеть, что $\phi-$ гомоморфизм. Пусть $f=\psi \phi: M \rightarrow M$. Тогда для каждого $x \in X$ имеем

$$
f p(x)=\psi \phi(p(x))=\psi(g(x)+g(\operatorname{Ker}(p))=p g(x) .
$$

Следовательно, $f p=p g$. 
Следствие 4. Пусть $p: X \rightarrow M-$ эпиморфное $\mathscr{X}$-накрытие модуля $M$. Следующие условия равносильны:

(1) $M-\mathscr{X}$-идемпотентно коинвариантный модуль;

(2) если $X=\bigoplus_{I} X_{i}$, то

$$
\operatorname{Ker}(p)=\bigoplus_{I}\left(X_{i} \cap \operatorname{Ker}(p)\right) ;
$$

(3) если $X=X_{1} \oplus X_{2}$, то

$$
\operatorname{Ker}(p)=\left(X_{1} \cap \operatorname{Ker}(p)\right) \oplus\left(X_{2} \cap \operatorname{Ker}(p)\right) ;
$$

(4) если $e \in \operatorname{End}(X)$ - идемпотент, то $\operatorname{Ker}(p)=e(\operatorname{Ker}(p)) \oplus(1-e)(\operatorname{Ker}(p))$.

Лемма 5. Пусть $M-$ модуль и $N-$ прямое слагаемое $M$. Если $M-\mathscr{X}$ идемпотентно коинвариантный модуль и $N$ обладает $\mathscr{X}$-накрытием, то модуль $N$ является $\mathscr{X}$-идемпотентно коинвариантным.

ДоказАтеЛЬСтво. Пусть $p: X \rightarrow M$ и $p_{1}: X_{1} \rightarrow N-\mathscr{X}$-накрытия, $\pi: M \rightarrow N-$ проекция и $\iota: N \rightarrow M-$ вложение. Рассмотрим произвольный идемпотентный эндоморфизм $g_{1}$ модуля $X_{1}$. Существуют такие гомоморфизмы $h_{1}: X_{1} \rightarrow X$ и $h_{2}: X \rightarrow X_{1}$, что $p h_{1}=\iota p_{1}$ и $p_{1} h_{2}=\pi p$. Следовательно, $p_{1} h_{2} h_{1}=p_{1}$ и $h_{2} h_{1}$ - изоморфизм. Тогда для некоторого гомоморфизма $h: X_{1} \rightarrow X_{1}$ имеем $h\left(h_{2} h_{1}\right)=\operatorname{id}_{X_{1}}$. Пусть $g=h_{1}\left(g_{1} h\right) h_{2}: X \rightarrow X$. Тогда $g-$ идемпотентный эндоморфизм модуля $X$. Так как $M-\mathscr{X}$-идемпотентно коинвариантный модуль, существует такой гомоморфизм $f: M \rightarrow M$, что $f p=p g$. Пусть $f_{1}=\pi f \iota$. Тогда

$$
f_{1} p_{1}=\pi f \iota p_{1}=\pi f p h_{1}=\pi p g h_{1}=\pi p h_{1}\left(g_{1} h\right) h_{2} h_{1}=\pi p h_{1} g_{1}=p_{1} h_{2} h_{1} g_{1}=p_{1} g_{1} .
$$

Таким образом, $N-\mathscr{X}$-идемпотентно коинвариантный модуль.

Пусть $M_{1}, M_{2}$ - правые $R$-модули. Модуль $M_{1}$ называется $\mathscr{X}$ - $M_{2}$-nроективным, если существуют $\mathscr{X}$-накрытия $p_{1}: X_{1} \rightarrow M_{1}, p_{2}: X_{2} \rightarrow M_{2}$ такие, что для любого гомоморфизма $g: X_{1} \rightarrow X_{2}$ существует гомоморфизм $f: M_{1} \rightarrow M_{2}$, для которого коммутативна диаграмма

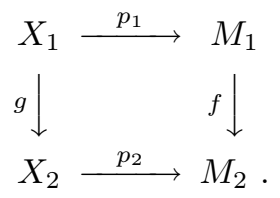

Если модуль $M \mathscr{X}$ - $M$-проективен, то $M$ называется $\mathscr{X}$-эндоморфизм-коинвариантным. Два правых $R$-модуля $M_{1}$ и $M_{2}$ называются взаимно $\mathscr{X}$-nроективными, если $M_{1} \mathscr{X}-M_{2}$-проективен и $M_{2} \mathscr{X}$ - $M_{1}$-проективен.

Лемма 6. Пусть $M_{1}, M_{2}$ - взаимно $\mathscr{X}$-проективные правые $R$-модули и $p_{1}: X_{1} \rightarrow M_{1}, p_{2}: X_{2} \rightarrow M_{2}$ - эпиморфные $\mathscr{X}$-накрытия. Если $X_{1} \simeq X_{2}$, то $M_{1} \simeq M_{2}$.

ДокаЗАТЕЛЬСтво. Пусть $g: X_{1} \rightarrow X_{2}$ - изоморфизм. По предположению существуют такие гомоморфизмы $f_{1}: M_{1} \rightarrow M_{2}$ и $f_{2}: M_{2} \rightarrow M_{1}$, что $f_{1} p_{1}=p_{2} g$ и $f_{2} p_{2}=p_{1} g^{-1}$. Тогда $f_{1} f_{2} p_{2}=p_{2}$ и $f_{2} f_{1} p_{1}=p_{1}$. Следовательно, $f_{1} f_{2}=\mathrm{id}_{M_{2}}$ и $f_{2} f_{1}=\operatorname{id}_{M_{1}}$. 
Предложение 7. Предположим, что модуль $M_{2}$ обладает эпиморфным $\mathscr{X}$-накрытием $p_{2}: X_{2} \rightarrow M_{2}$ и каждый фактор-модуль $M_{1} / A$ модуля $M_{1}$ обладает таким $\mathscr{X}$-накрытием $p_{A}: X_{A} \rightarrow M_{1} / A$, что для всякого естественного гомоморфизма $f: M_{1} \rightarrow M_{1} / A$ существует расщепляющийся эпиморфизм $\psi: X_{1} \rightarrow X_{A}$, для которого выполнено равенство $p_{A} \psi=f p_{1}$, где $p_{1}: X_{1} \rightarrow M_{1}-$ $\mathscr{X}$-накрытие. Тогда если модуль $M_{2} M_{1}$ - $\mathscr{X}$-проективен, то $M_{2} M_{1}$-проективен.

ДокАЗАТЕльство. Пусть $A-$ подмодуль модуля $M=M_{1} \oplus M_{2}$, для которого выполнено равенство $M=A+M_{1}$. Несложно заметить, что существует такой гомоморфизм $g: M_{2} \rightarrow M_{1} /\left(A \cap M_{1}\right)$, что $g\left(m_{2}\right)=m_{1}+A \cap M_{1}$, если $a=m_{1}+m_{2}$, где $m_{1} \in M_{1}, m_{2} \in M_{1}$ и $a \in A$. Согласно предположению для некоторого эпиморфизма $\psi: X_{1} \rightarrow X_{1}^{\prime}$ имеет место коммутативная диаграмма

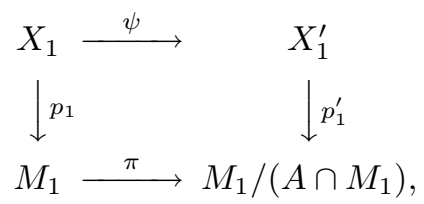

где $p_{1}^{\prime}: X_{1}^{\prime} \rightarrow M_{1} /\left(A \cap M_{1}\right)-\mathscr{X}$-оболочка, $\pi$ - естественный гомоморфизм и $\psi \iota=1_{X_{1}^{\prime}}$ для некоторого гомоморфизма $\iota: X_{1}^{\prime} \rightarrow X_{1}$.

Из определения $\mathscr{X}$-накрытия следует существование гомоморфизма $f$ : $X_{2} \rightarrow X_{1}^{\prime}$, для которого коммутативна диаграмма



Поскольку модуль $M_{2} \mathscr{C}$ - $M_{1}$-проективен, существует гомоморфизм $\phi: M_{2} \rightarrow$ $M_{1}$, для которого коммутативна диаграмма

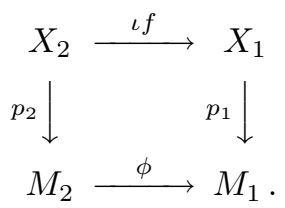

Для любого $b \in M_{2}$ существует такой элемент $x \in X_{2}$, что $b=p_{2}(x)$. Тогда

$$
\begin{gathered}
\phi(b)=\phi p_{2}(x)=p_{1} \iota f(x), \\
g(b)=g p_{2}(x)=p_{1}^{\prime} f(x)=p_{1}^{\prime} \psi \iota f(x)=\pi p_{1} \iota f(x)=\pi \phi(b) .
\end{gathered}
$$

Пусть

$$
C=\left\{\phi\left(m_{2}\right)+m_{2} \mid m_{2} \in M_{2}\right\} \leq M .
$$

Тогда $M=M_{1} \oplus C$ и $C \leq A$. Следовательно, согласно [15, 4.12] модуль $M_{2}$ $M_{1}$-проективен.

Предложение 8. Пусть $p_{1}: X_{1} \rightarrow M_{1}$ и $p_{2}: X_{2} \rightarrow M_{2}$ - эпиморфные $\mathscr{X}$-накрытия и $\operatorname{Ker}\left(p_{1}\right) \ll X_{1}$. Если модуль $M_{1}$ является $M_{2}$-проективным, то модуль $M_{1} M_{2}$ - $\mathscr{X}$-проективен.

ДокАЗАтЕЛЬСтво. Пусть $f: X_{1} \rightarrow X_{2}$ - гомоморфизм модулей. Без ограничения общности можно считать, что $M_{2}=X_{2} / \operatorname{Ker}\left(p_{2}\right)$ и $p_{2}: X_{2} \rightarrow$ $X_{2} / \operatorname{Ker}\left(p_{2}\right)$ - естественный гомоморфизм. Положим $N=\operatorname{Ker}\left(p_{2}\right)+f\left(\operatorname{Ker}\left(p_{1}\right)\right)$. 
Так как $f\left(\operatorname{Ker}\left(p_{1}\right)\right) \subseteq N$, для некоторого гомоморфизма $f_{1}: M_{1} \rightarrow X_{2} / N$ имеет место равенство $\pi p_{2} f=f_{1} p_{1}$, где $\pi: X_{2} / \operatorname{Ker}\left(p_{2}\right) \rightarrow X_{2} / N-$ естественный гомоморфизм. Согласно предположению для некоторого гомоморфизма $f_{2}: M_{1} \rightarrow M_{2}$ имеем $\pi f_{2}=f_{1}$. Из определения $\mathscr{X}$-накрытия следует существование гомоморфизма $g: X_{1} \rightarrow X_{2}$, для которого выполнено равенство $p_{2} g=f_{2} p_{1}$. Тогда для произвольного элемента $x \in X_{1}$ существуют такие элементы $x_{1} \in \operatorname{Ker}\left(p_{1}\right), x_{2} \in \operatorname{Ker}\left(p_{2}\right)$, что $(f-g)(x)=x_{2}+f\left(x_{1}\right)$. Поскольку

$$
p_{2}(f-g)\left(x-x_{1}\right)=p_{2}\left(x_{2}+f\left(x_{1}\right)\right)-p_{2} f\left(x_{1}\right)=0,
$$

то $x \in \operatorname{Ker}\left(p_{1}\right)+\operatorname{Ker}\left(p_{2}(f-g)\right)$. Таким образом,

$$
X_{1}=\operatorname{Ker}\left(p_{1}\right)+\operatorname{Ker}\left(p_{2}(f-g)\right)=\operatorname{Ker}\left(p_{2}(f-g)\right) .
$$

Следовательно, $(f-g)\left(X_{1}\right) \subseteq \operatorname{Ker}\left(p_{2}\right)$ и поскольку $g\left(\operatorname{Ker}\left(p_{1}\right)\right) \leq \operatorname{Ker}\left(p_{2}\right)$, то $f\left(\operatorname{Ker}\left(p_{1}\right)\right) \leq \operatorname{Ker}\left(p_{2}\right)$. Тогда для некоторого гомоморфизма $f^{\prime}: M_{1} \rightarrow M_{2}$ выполнено равенство $p_{2} f=f^{\prime} p_{1}$.

Важным следствием леммы 6 и предложения 7 является

Следствие 9 [27]. Пусть $M, N$ - правые $R$-модули и $\pi_{1}: P \rightarrow M, \pi_{2}: P^{\prime} \rightarrow$ $N$ - проективные накрытия модулей $M$ и $N$ соответственно. Тогда следующие условия равносильны:

(1) модуль $M N$-проективен;

(2) для каждого гомоморфизма $f: P \rightarrow P^{\prime}$ существует гомоморфизм $g$ : $M \rightarrow N$, для которого коммутативна диаграмма

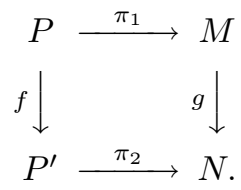

$B$ частности, если $\pi: P \rightarrow M-$ проективное накрытие модуля $M$, то модуль $M$ квазипроективен в точности тогда, когда $\operatorname{Ker}(\phi)$ является вполне инвариантным подмодулем модуля $P$.

Теорема 10. Пусть $M=M_{1} \oplus M_{2}-$ модуль и $p_{1}: X_{1} \rightarrow M_{1}, p_{2}: X_{2} \rightarrow M_{2}$, $p_{1} \oplus p_{2}: X_{1} \oplus X_{2} \rightarrow M-\mathscr{X}$-накрытия правых $R$-модулей. Если модуль $M \mathscr{X}$ идемпотентно коинвариантен, то $M_{i} \mathscr{X}-M_{j}$-проективен для каждого $i \neq j$.

ДокаЗАтЕЛЬСтво. Пусть $g: X_{1} \rightarrow X_{2}$ - гомоморфизм. Определим гомоморфизм $g^{\prime}: X_{1} \oplus X_{2} \rightarrow X_{1} \oplus X_{2}$ с помощью матрицы

$$
g^{\prime}=\left(\begin{array}{cc}
\operatorname{id}_{X_{1}} & 0 \\
g & 0
\end{array}\right) .
$$

Тогда $g^{\prime 2}=g^{\prime}$. Так как $M-\mathscr{X}$-идемпотентно коинвариантный модуль, для некоторого $f^{\prime} \in \operatorname{End}(M)$ выполнено равенство $f^{\prime} p=p g^{\prime}$. Пусть $f=\pi_{2} f^{\prime} \iota_{1}$, где $\iota_{1}: M_{1} \rightarrow M-$ естественное вложение и $\pi_{2}: M \rightarrow M_{2}$ - каноническая проекция. Тогда $f p_{1}=p_{2} g$. Таким образом, модуль $M_{1} \mathscr{X}-M_{2}$-проективен.

Теорема 11. Пусть $M=\bigoplus_{i=1}^{n} M_{i}-$ модуль и $p_{i}: X_{i} \rightarrow M_{i}-\mathscr{X}$-накрытия. Тогда следующие условия равносильны:

(1) $M_{1} \oplus M_{2} \oplus \cdots \oplus M_{n}-\mathscr{X}$-эндоморфизм-коинвариантный модуль; 
$(2)$ модули $M_{i}$ и $M_{j}$ взаимно $\mathscr{X}$-проективны для каждых $i, j \in\{1,2, \ldots, n\}$. ДокАЗАТЕЛЬСтво. Достаточно рассмотреть случай, когда $n=2$.

$(1) \Rightarrow(2)$ Так как каждый $\mathscr{X}$-эндоморфизм-коинвариантный модуль является $\mathscr{X}$-идемпотентно коинвариантным модулем, п. (2) следует из леммы 5 и теоремы 10.

$(2) \Rightarrow(1)$ Предположим, что $M_{i}-\mathscr{X}-M_{j}$-проективный модуль для каждых $i, j \in\{1,2\}$. Согласно [24, предложение 5.5.4] $p_{1} \oplus p_{2}: X_{1} \oplus X_{2} \rightarrow M_{1} \oplus M_{2}-\mathscr{X}$ накрытие. Пусть $g$ - эндоморфизм модуля $X_{1} \oplus X_{2}, \iota_{1}: X_{1} \rightarrow X_{1} \oplus X_{2}, \iota_{2}: X_{2} \rightarrow$ $X_{1} \oplus X_{2}$ - вложения и $\pi_{1}: X_{1} \oplus X_{2} \rightarrow X_{1}, \pi_{2}: X_{1} \oplus X_{2} \rightarrow X_{2}$ - канонические проекции. Так как модули $M_{i}$ и $M_{j}$ взаимно $\mathscr{X}$-проективны для каждых $i, j \in$ $\{1,2\}$, существует такой гомоморфизм $f_{j i}: M_{i} \rightarrow M_{j}$, что $p_{j}\left(\pi_{j} g \iota_{i}\right)=f_{j i} p_{i}$. Пусть $f: M_{1} \oplus M_{2} \rightarrow M_{1} \oplus M_{2}$ - эндоморфизм, у которого матрица имеет вид $\left(\begin{array}{ll}f_{11} & f_{12} \\ f_{21} & f_{22}\end{array}\right)$. Тогда $\left(p_{1} \oplus p_{2}\right) g=f\left(p_{1} \oplus p_{2}\right)$. Таким образом, $M=M_{1} \oplus M_{2}-$ $\mathscr{X}$-эндоморфизм-коинвариантный модуль.

Следствие 12. Модуль $M \mathscr{X}$-эндоморфизм-коинвариантен в точности тогда, когда $M \oplus M-\mathscr{X}$-эндоморфизм-коинвариантный модуль.

Следствие 13. Пусть $M=\bigoplus_{i=1}^{n} M_{i}-$ правый модуль над совершенным справа кольцом. Следующие условия равносильны:

(1) $M_{1} \oplus M_{2} \oplus \cdots \oplus M_{n}-$ квазипроективный модуль;

(2) модули $M_{i}$ и $M_{j}$ взаимно проективны для каждых $i, j \in\{1,2, \ldots, n\}$.

Следствие 14. Если $M$ - правый модуль над совершенным справа кольцом, то $M$ является квазипроективным модулем в точности тогда, когда $M \oplus$ $M$ - квазипроективный модуль.

Модуль $M$ называется чисто бесконечным, если $M=M \oplus M$. Если модуль $M$ не изоморфен собственному прямому слагаемому, то он называется прямо конечным.

Теорема 15. Пусть $M-\mathscr{X}$-идемпотентно коинвариантный модуль и $p$ : $X \rightarrow M-$ эпиморфное $\mathscr{X}$-накрытие модуля $M$. Следующие условия равносильны:

(1) модуль $M$ чисто бесконечен в точности тогда, когда $X$ - чисто бесконечный модуль;

(2) если $X-$ прямо конечный модуль, то модуль $M$ прямо конечный;

(3) если $\mathscr{X}-$ класс проективных модулей и модуль $M$ не прямо конечный, то для модуля $M$ имеет место разложение $M=M_{1} \oplus M_{2} \oplus M_{3}$, где $M_{1} \cong M_{2} \neq 0$.

ДокАЗАТЕЛЬСТво. (1) $(\Rightarrow)$ Предположим, что модуль $M$ бесконечный. Тогда $M=M_{1} \oplus M_{2}$, где $M_{1} \simeq M_{2} \simeq M$. Пусть $p_{1}: X_{1} \rightarrow M_{1}$ и $p_{2}: X_{2} \rightarrow M_{2}-$ $\mathscr{X}$-оболочки. Ясно, что $X \simeq X_{1} \oplus X_{2}$ и $X \simeq X_{1} \simeq X_{2}$. Таким образом, модуль $X$ чисто бесконечный.

$(\Leftarrow)$ Предположим, что имеет место разложение $X=X_{1} \oplus X_{2}$ и $X_{1} \simeq X_{2} \simeq$ $X$. Тогда $X_{1}=e(X)$ и $X_{2}=(1-e)(X)$ для некоторого $e^{2}=e \in \operatorname{End}(X)$. Согласно следствию $4 \operatorname{Ker}(p)=e(\operatorname{Ker}(p)) \oplus(1-e)(\operatorname{Ker}(p))$. Тогда

$$
\frac{X}{\operatorname{Ker}(p)} \simeq \frac{X_{1}}{e(\operatorname{Ker}(p))} \oplus \frac{X_{2}}{(1-e)(\operatorname{Ker}(p))} .
$$

Обозначим $M_{1}=\frac{X_{1}}{e(\operatorname{Ker}(p))}$ и $M_{2}=\frac{X_{2}}{(1-e)(\operatorname{Ker}(p))}$. Покажем, что естественные гомоморфизмы $p_{1}: X_{1} \rightarrow M_{1}$ и $p_{2}: X_{2} \rightarrow M_{2}$ являются $\mathscr{X}$-оболочками. Пусть 
$\iota: M_{1} \rightarrow M_{1} \oplus M_{2}, \iota^{\prime}: X_{1} \rightarrow X_{1} \oplus X_{2}-$ естественные вложения и $\pi: M_{1} \oplus M_{2} \rightarrow$ $M_{1}, \pi^{\prime}: X_{1} \oplus X_{2} \rightarrow X_{1}$ - проекции. Рассмотрим произвольный гомоморфизм $f:$ $U \rightarrow M_{1}$, где $U \in \mathscr{X}$. Поскольку $p_{1} \oplus p_{2}: X_{1} \oplus X_{2} \rightarrow M_{1} \oplus M_{2}-\mathscr{X}$-оболочка, для некоторого гомоморфизма $g: U \rightarrow X_{1} \oplus X_{2}$ имеет место равенство $\left(p_{1} \oplus p_{2}\right) g=\iota f$ и, следовательно, $f=\pi \iota f=\pi\left(p_{1} \oplus p_{2}\right) \iota^{\prime} \pi^{\prime} g=p_{1} \pi^{\prime} g$. Предположим, что $\alpha p_{1}=p_{1}$ для некоторого гомоморфизма $\alpha: X_{1} \rightarrow X_{1}$. Тогда $\left(\alpha \oplus 1_{X_{2}}\right)\left(p_{1} \oplus p_{2}\right)=p_{1} \oplus p_{2}$. Из определения $\mathscr{X}$-оболочки следует, что $\alpha \oplus 1_{X_{2}}$ - изоморфизм и, следовательно, $\alpha$ также изоморфизм. Таким образом, $p_{1}: X_{1} \rightarrow M_{1}$ является $\mathscr{X}$-оболочкой. Аналогично показывается, что $p_{2}: X_{2} \rightarrow M_{2}-\mathscr{X}$-оболочка. По теореме 10 модули $M_{1}$ и $M_{2}$ взаимно $\mathscr{X}$-проективны. Тогда из леммы 6 следует, что $M_{1} \simeq$ $M_{2} \simeq M$. Таким образом, модуль $M$ чисто бесконечен.

(2) Предположим, что $M$ не прямо конечный. Тогда $M=M_{1} \oplus M_{2}$, где $M_{1} \simeq M, M_{2} \neq 0$. Несложно заметить, что $X \simeq X_{1} \oplus X_{2}$ и $X_{1} \simeq X$. Таким образом, модуль $X$ не прямо конечный.

(3) Из п. (2) следует, что модуль $X$ не прямо конечный. Тогда существуют подмодули $X_{1}, X_{2}$ и $X_{3}$ модуля $X$, для которых имеет место разложение $X=$ $X_{1} \oplus X_{2} \oplus X_{3}, X_{1} \neq 0$ и $X_{1} \simeq X_{2}$. Для некоторых ортогональных идемпотентов $\left\{e_{1}, e_{2}, e_{3}\right\}$ из кольца $\operatorname{End}(X)$ имеют место равенства $X_{1}=e_{1}(X), X_{2}=e_{2}(X)$, $X_{3}=e_{3}(X)$. Согласно следствию 4

$$
\operatorname{Ker}(p)=e_{1}(\operatorname{Ker}(p)) \oplus e_{2}(\operatorname{Ker}(p)) \oplus e_{3}(\operatorname{Ker}(p)) .
$$

Тогда

$$
\frac{X}{\operatorname{Ker}(p)} \simeq \frac{X_{1}}{e_{1}(\operatorname{Ker}(p))} \oplus \frac{X_{2}}{e_{2}(\operatorname{Ker}(p))} \oplus \frac{X_{3}}{e_{3}(\operatorname{Ker}(p))} .
$$

Обозначим $M_{1}=\frac{X_{1}}{e(\operatorname{Ker}(p))}, M_{2}=\frac{X_{2}}{(1-e)(\operatorname{Ker}(p))}$ и $M_{3}=\frac{X_{3}}{e_{3}(\operatorname{Ker}(p))}$. Тогда $M \simeq$ $M_{1} \oplus M_{2} \oplus M_{3}$. Так как $\pi_{i}: X_{i} \rightarrow X_{i} / e_{i}(\operatorname{Ker}(p))-\mathscr{X}$-оболочка для каждого $1 \leq i \leq 3$, то $M_{1} \simeq M_{2}$.

Пусть $M$ - правый $R$-модуль. Модуль $M$ называется $\mathscr{X}$-модулем со свойством подбема, если существует такое $\mathscr{X}$-накрытие $p: X \rightarrow M$ модуля $M$, что для любого идемпотента $g \in \operatorname{End}(X)$ найдется идемпотент $f: M \rightarrow M$, для которого выполнено равенство $g(X)+\operatorname{Ker}(p)=p^{-1}(f(M))$.

Следующее утверждение проверяется непосредственно.

Предложение 16. Пусть $p: X \rightarrow M-$ эпиморфное $\mathscr{X}$-накрытие. Если $M-\mathscr{X}$-идемпотентно коинвариантный модуль, то $M-\mathscr{X}$-модуль со свойством подъема.

Предложение 17. Пусть $N-$ прямое слагаемое модуля $M$. Если модуль $M$ является $\mathscr{X}$-модулем со свойством подъема, обладающим эпиморфным $\mathscr{X}$-накрытием, и модуль $N$ обладает $\mathscr{X}$-накрытием, то $N-\mathscr{X}$-модуль со свойСтвом Подъема.

ДоказатеЛьство. Пусть $p_{1}: X_{1} \rightarrow N-\mathscr{X}$-накрытие. Легко видеть, что $X_{1}$ изоморфно такому прямому слагаемому $K$ модуля $X$, что $\left.p\right|_{K}: K \rightarrow N-\mathscr{X}$ накрытие $N$. Таким образом, можно предполагать, что $p_{1}=\left.p\right|_{X_{1}}: X_{1} \rightarrow N-$ $\mathscr{X}$-накрытие $N$ и $X_{1}-$ прямое слагаемое модуля $X$. Пусть $g: X_{1} \rightarrow X_{1}-$ идемпотентный эндоморфизм модуля $X_{1}$. Рассмотрим гомоморфизм $g^{\prime}=\iota g \pi$ : $X \rightarrow X$, где $\iota: X_{1} \rightarrow X$ и $\pi: X \rightarrow X_{1}-$ вложения. Тогда $g^{\prime 2}=g^{\prime}$. Так как модуль $M$ является $\mathscr{X}$-модулем со свойством подъема, существует такой гомоморфизм $f^{\prime 2}=f^{\prime}: M \rightarrow M$, что $g^{\prime}(X)+\operatorname{Ker}(p)=p^{-1}\left(f^{\prime}(M)\right)$. Тогда 
$f^{\prime}(M)=p\left(g\left(X_{1}\right)\right)=p_{1}\left(g\left(X_{1}\right)\right) \leq N$ и, следовательно, $f^{\prime}(M)$ - прямое слагаемое модуля $N$. Существует такой идемпотентный гомоморфизм $f: N \rightarrow N$, что $p_{1}\left(g\left(X_{1}\right)\right)=f^{\prime}(M)=f(N)$. Тогда $g\left(X_{1}\right)+\operatorname{Ker}\left(p_{1}\right)=p_{1}^{-1}(f(N))$. Таким образом, модуль $N$ является $\mathscr{X}$-модулем со свойством подъема.

Пусть $p: X \rightarrow M-\mathscr{X}$-накрытие модуля $M$ и $A-$ подмодуль модуля $M$. Подмодуль $A$ называется $\mathscr{X}$-козамкнутым в $M$, если для некоторого идемпотентного эндоморфизма $g \in \operatorname{End}(X)$ выполнено равенство $A=p(g(X))$.

Теорема 18. Пусть $p: X \rightarrow M-$ эпиморфное $\mathscr{X}$-накрытие. Следующие условия равносильны:

(1) $M-\mathscr{X}$-модуль со свойством подъема;

(2) каждый $\mathscr{X}$-козамкнутый подмодуль модуля $M$ является прямым слагаемым $M$.

ДокаЗАтельство. (1) $\Rightarrow(2)$ Пусть $U=p(g(X))$, где $g^{2}=g \in \operatorname{End}(X)$. Существует такой эндоморфизм $f^{2}=f \in \operatorname{End}(M)$, что $g(X)+\operatorname{Ker}(p)=p^{-1}(f(M))$. Следовательно, $U=p(g(X))=f(M)$ - прямое слагаемое $M$.

$(2) \Rightarrow(1)$ Пусть $g$ - идемпотентный элемент из кольца $\operatorname{End}(X)$. Согласно предположению $U=p(g(X))$ - прямое слагаемое модуля $M$. Существует гомоморфизм $f^{2}=f \in \operatorname{End}(M)$, для которого выполнено равенство $p(g(X))=$ $f(M)$. Тогда $g(X)+\operatorname{Ker}(p)=p^{-1}(f(M))$. Таким образом, модуль $M$ является $\mathscr{X}$-модулем со свойством подъема.

Если $\mathscr{X}-$ класс проективных правых $R$-модулей, то с помощью стандартных рассуждений можно показать, что над совершенным справа кольцом $R$ правый модуль $M$ является $\mathscr{X}$-модулем со свойством подъема в точности тогда, когда $M$ - модуль со свойством подъема [28, теорема 2.6].

До конца настоящего раздела будем предполагать, что все рассматриваемые модули $M$ обладают $\mathscr{C}$-накрытиями $p: X \rightarrow M$, где $\mathscr{C}$ - некоторый класс модулей, удовлетворяющий следующим условиям:

(1) класс модулей $\mathscr{C}$ замкнут относительно изоморфизмов;

(2) каждый фактор-модуль $M / A$ модуля $M$ обладает эпиморфным $\mathscr{C}$-накрытием $p_{M / A}: X_{M / A} \rightarrow M / A$, у которого $\operatorname{Ker}\left(p_{M / A}\right) \ll X_{M / A}$;

(3) для каждого прямого слагаемого $N$ модуля $M$ и каждого естественного гомоморфизма $\pi: N \rightarrow N / A$ существует расщепляющийся эпиморфизм $\psi$ : $X_{N} \rightarrow X_{N / A}$, для которого коммутативна диаграмма

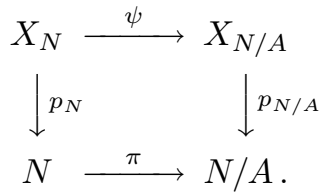

Модуль $M$ называется SSP-модулем, если сумма двух прямых слагаемых модуля $M$ является прямым слагаемым модуля $M$.

Предложение 19. Пусть $M=M_{1} \oplus M_{2}$ - модуль и $p_{i}: X_{i} \rightarrow M_{i}, i=1,2$, $p=p_{1} \oplus p_{2}: X_{1} \oplus X_{2} \rightarrow M-\mathscr{C}$-накрытия. Если $X-\mathrm{SSP}$-модуль и каждый $\mathscr{C}$-козамкнутый подмодуль $N \leq M$, для которого либо $N+M_{1}=M$, либо $N+M_{2}=M$, является прямым слагаемым модуля $M$, то $M-\mathscr{C}$-модуль со свойством подъема.

ДокАЗАтЕльСтво. Пусть $X-\mathrm{SSP}$-модуль и $N=p(g(X))-\mathscr{C}$-козамкнутый подмодуль модуля $M$, где $g$ - идемпотент из кольца $\operatorname{End}(X)$. Тогда подмодуль $H=g(X)+X_{2}$ является прямым слагаемым модуля $X$. Следовательно, 
$p(H)-\mathscr{C}$-козамкнутый подмодуль модуля $M$. С другой стороны, $X=H+X_{1}$ и тем самым $M=p(H)+M_{1}$. По предположению $p(H)$ - прямое слагаемое модуля $M$. Тогда для некоторого подмодуля $H^{\prime}$ модуля $M$ имеет место равенство $M=p(H) \oplus H^{\prime}$. Несложно заметить, что $H^{\prime}=p\left(X^{\prime}\right)$ для некоторого такого прямого слагаемого $X^{\prime}$ модуля $X$, что $\left.p\right|_{X^{\prime}}: X^{\prime} \rightarrow H^{\prime}-\mathscr{C}$-накрытие. Так как $X^{\prime}+g(X)-$ прямое слагаемое модуля $X$, то $p g(X) \oplus H^{\prime}=p\left(g(X)+X^{\prime}\right)-\mathscr{C}$ козамкнутый подмодуль модуля $M$. Поскольку $M=p(g(X))+p\left(X_{2}\right)+H^{\prime}$, то $M=\left[p g(X) \oplus H^{\prime}\right]+M_{2}$. Следовательно, $p g(X) \oplus H^{\prime}$ - прямое слагаемое $M$. Таким образом, $N=p g(X)$ - прямое слагаемое $M$.

Теорема 20. Следующие условия эквивалентны для модуля $M$ :

(1) $M-\mathscr{C}$-идемпотентно коинвариантный модуль;

(2) $M-\mathscr{C}$-модуль со свойством подъема и для каждого разложения $M=$ $M_{1} \oplus M_{2}$ модули $M_{1}$ и $M_{2}$ взаимно $\mathscr{C}$-проективны;

(3) $M-\mathscr{C}$-модуль со свойством подъема и для каждого разложения $M=$ $M_{1} \oplus M_{2}$ модули $M_{1}$ и $M_{2}$ взаимно проективны.

ДокАзАтЕльство. $(1) \Rightarrow(2)$ Импликация следует из теоремы 10 и предложения 16.

$(2) \Rightarrow(3)$ Следует из предложения 7.

$(3) \Rightarrow(1)$ Предположим, что $p: X \rightarrow M-$ эпиморфное $\mathscr{C}$-накрытие модуля $M$. Пусть $g$ - идемпотент из кольца $\operatorname{End}(X)$. Поскольку модуль $M$ является $\mathscr{C}$-модулем со свойством подъема, подмодули $A=p(g(X)), B=p((1-g)(X)$ являются прямыми слагаемыми модуля $M$. Тогда $A+B=M$ и $M=B \oplus B^{\prime}$ для некоторого $B^{\prime} \leq M$. Так как модуль $A B$-проективен, существует такой подмодуль $C \leq A$, что $M=B \oplus C$. Пусть $\pi: B \oplus C \rightarrow C$ - каноническая проекция. Для любого $x \in X$ существуют такие элементы $x_{1}, y_{1}, y_{2} \in X$, что $p g\left(y_{1}\right)+p(1-g)\left(y_{2}\right) \in C$ и

$$
p(x)=p(1-g)\left(x_{1}\right)+p g\left(y_{1}\right)+p(1-g)\left(y_{2}\right)=p g(x)+p(1-g)(x) .
$$

Тогда

$$
\begin{aligned}
0=p(1-g)\left(x_{1}\right)+p & (1-g)\left(y_{2}\right)-p(1-g)(x)+p g\left(y_{1}\right)-p g(x) \\
& =p\left[(1-g)\left(x_{1}\right)+(1-g)\left(y_{2}\right)-(1-g)(x)+g\left(y_{1}\right)-g(x)\right] .
\end{aligned}
$$

Таким образом,

$$
(1-g)\left(x_{1}\right)+(1-g)\left(y_{2}\right)-(1-g)(x)+g\left(y_{1}\right)-g(x) \in \operatorname{Ker}(p) .
$$

Следовательно, $g\left(y_{1}\right)-g(x) \in g(\operatorname{Ker}(p))$. Пусть $a \in \operatorname{Ker}(p)-$ элемент, для которого выполнено равенство $g\left(y_{1}\right)-g(x)=g(a)$. Так как $p g\left(y_{1}\right)+p(1-g)\left(y_{2}\right) \in$ $C \leq A=p g(X)$, то $(1-g)\left(y_{2}\right) \in(1-g)(\operatorname{Ker}(p))$ и тем самым $(1-g)\left(y_{2}\right)=(1-g)(b)$ для некоторого $b \in \operatorname{Ker}(p)$. Тогда

$$
\begin{array}{r}
(\pi p-p g)(x)=p g\left(y_{1}\right)+p(1-g)\left(y_{2}\right)-p g(x)=p\left[g\left(y_{1}\right)-g(x)\right]+p(1-g)\left(y_{2}\right) \\
=p(g(a))+p(1-g)(b)=(\pi p-p g)(-a)+(\pi p-p g)(b) .
\end{array}
$$

Следовательно, $X=\operatorname{Ker}(p)+\operatorname{Ker}(\pi p-p g)$. Поскольку $\operatorname{Ker}(p) \ll X$, то $\pi p-p g=0$. Таким образом, модуль $M \mathscr{C}$-идемпотентно коинвариантен.

Следствие 21. Пусть $R$ - совершенное справа кольцо. Если $M-$ правый $R$-модуль, то следующие утверждения равносильны:

(1) $M-$ квазидискретный модуль;

(2) $M$ - модуль со свойством подъема и для каждого разложения $M=$ $M_{1} \oplus M_{2}$ модули $M_{1}$ и $M_{2}$ взаимно проективны. 


\section{3. $\mathscr{X}$-дискретные и $\mathscr{X}$-непрерывные модули}

Пусть $M-$ правый $R$-модуль, $p: X \rightarrow M-\mathscr{X}$-накрытие модуля $M, S=$ $\operatorname{End}(X)$. Если для некоторых гомоморфизмов $g_{1}, g_{2} \in S, f \in \operatorname{End}(M)$ имеет место равенство $p g_{1}=f p=p g_{2}$, то для каждого $h \in S$ имеем $p\left(g_{1}-g_{2}\right) h=0$ и, следовательно, $p=p\left(1-\left(g_{1}-g_{2}\right) h\right)$. Тогда $1-\left(g_{1}-g_{2}\right) h$ является автоморфизмом. Таким образом, $g_{1}-g_{2} \in J(S)$ и тем самым определен кольцевой гомоморфизм $\Phi: \operatorname{End}(M) \rightarrow S / J(S)$, действующий по правилу $\Phi(f)=f^{\prime}+J(S)$, где $f^{\prime}:$ $X \rightarrow X-$ гомоморфизм, для которого выполнено равенство $p f^{\prime}=f p$. Ядро гомоморфизма $\Phi$ будем обозначать через $\nabla(M)=\operatorname{Ker}(\Phi)$. Тогда имеет место вложение $\bar{\Phi}: M / \nabla(M) \rightarrow S / J(S)$. Несложно заметить, что если $\mathscr{X}-$ класс проективных правых $R$-модулей, то $\nabla(M)=\{f \in \operatorname{End}(M) \mid f(M) \ll M\}$.

Лемма 22. Пусть $R$ - совершенное справа кольцо, $M$ - квазидискретный правый $R$-модуль и $p: P \rightarrow M-$ проективное накрытие модуля $M$. Следующие условия равносильны:

(1) $M$ - дискретный модуль;

(2) если для идемпотентов $e_{1}, e_{2} \in \operatorname{End}(P), e_{1}^{\prime}, e_{2}^{\prime} \in \operatorname{End}(M)$ выполнены равенства $p_{i}=e_{i}^{\prime} p, i=1,2$, для гомоморфизмов $\alpha, \alpha^{\prime}$ коммутативна диаграмма

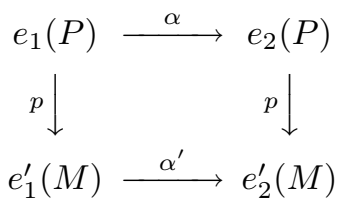

и $\alpha$ является изоморфизмом, то $\alpha^{\prime}-$ изоморфизм.

ДокаЗАтельство. (1) $\Rightarrow(2)$ Пусть $M$ - дискретный модуль, $e_{1}, e_{2} \in$ $\operatorname{End}(P), e_{1}^{\prime}, e_{2}^{\prime} \in \operatorname{End}(M)$ - идемпотенты, $\alpha: e_{1}(P) \rightarrow e_{2}(P)-$ изоморфизм, $\alpha: e_{1}^{\prime}(M) \rightarrow e_{2}^{\prime}(M)$ - гомоморфизм и выполнены равенства $p e_{i}=e_{i}^{\prime} p, i=1,2$, $p \alpha=\alpha^{\prime} p$. Ясно, что $\alpha^{\prime}-$ эпиморфизм. Поскольку модуль $M$ дискретный, $e_{1}^{\prime}(M)=\operatorname{Ker}\left(\alpha^{\prime}\right) \oplus N$, где $N \leq M$. Так как $p_{\mid e_{1}(P)}^{-1}\left(\operatorname{Ker}\left(\alpha^{\prime}\right)\right)=\operatorname{Ker}(p \alpha)-$ малый подмодуль модуля $e_{1}(P)$, то $\operatorname{Ker}\left(\alpha^{\prime}\right)=0$.

$(2) \Rightarrow(1)$ Предположим, что для квазидискретного модуля $M$ выполнены условия п. (2). Пусть $e \in \operatorname{End}(M)$ и $f: M \rightarrow e M-$ эпиморфизм. Рассмотрим проективную оболочку $p^{\prime}: P^{\prime} \rightarrow e M$ модуля $e M$. Для некоторого гомоморфизма $\alpha: P \rightarrow P^{\prime}$ имеет место равенство $p^{\prime} \alpha=f p$. Несложно заметить, что гомоморфизм $\alpha$ является эпиморфизмом. Тогда имеет место равенство $P=P_{0} \oplus \operatorname{Ker}(\alpha)$, где $P_{0} \leq P$. Поскольку $M$ - квазидискретный модуль, то $M=p\left(P_{0}\right) \oplus p(\operatorname{Ker}(\alpha))$. Из условия п. (2) следует, что $f_{\mid p\left(P_{0}\right)}$ является изоморфизмом. Таким образом, $f$ - расщепляющийся эпиморфимзм.

В настоящем разделе, если только не оговорено противное, будем предполагать, что $\mathscr{X}-$ класс правых $R$-модулей, замкнутый относительно изоморфизмов, $p: X \rightarrow M-$ эпиморфное $\mathscr{X}$-накрытие правого $R$-модуля $M$ и $S=\operatorname{End}(X)$ - полурегулярное кольцо. Согласно лемме 2 для каждого идемпотента $e^{2}=e \in \operatorname{End}(X)$ существует такой однозначно определенный идемпотент $f \in \operatorname{End}(M)$, что $p e=f p$. Далее этот идемпотент будем обозначать через $\hat{e}$.

Пусть $p: X \rightarrow M-\mathscr{X}$-накрытие модуля $M$. Модуль $M$ называется $\mathscr{X}$ дискретным, если выполнены следующие условия:

(1) $M-\mathscr{X}$-идемпотентно коинвариантный модуль; 
(2) если для идемпотентов $e_{1}, e_{2} \in \operatorname{End}(X)$ и гомоморфизмов $\alpha, \alpha^{\prime}$ коммутативна диаграмма

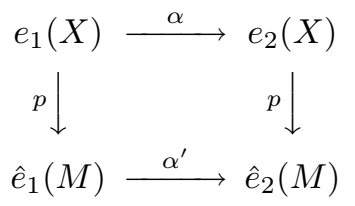

и $\alpha$ является изоморфизмом, то $\alpha^{\prime}-$ изоморфизм.

Лемма 23. Если $M-\mathscr{X}$-идемпотентно коинвариантный модуль, то все идемпотенты из кольца $\operatorname{End}(M) / \nabla(M)$ поднимаются по модулю идеала $\nabla(M)$.

ДокАзАтЕЛЬСтво. Пусть $s+\nabla(M)$ - идемпотент из кольца $\operatorname{End}(M) / \nabla(M)$. Тогда $\bar{\Phi}(s+\nabla(M))=s^{\prime}+J(S)$ - идемпотент из кольца $S / J(S)$, где $s p=p s^{\prime}$. Так как $S$ является полурегулярным кольцом, существует такой идемпотент $\varepsilon$ из $S$, что $s^{\prime}+J(S)=\varepsilon+J(S)$. Поскольку модуль $M \mathscr{X}$-идемпотент-коинвариантен, по лемме 2 существует идемпотент $e$ из кольца $\operatorname{End}(M)$, для которого выполнено равенство $\bar{\Phi}(e+\nabla(M))=\varepsilon+J(S)$. Следовательно, $s+\nabla(M)=e+\nabla(M)$.

Теорема 24. Если $M-\mathscr{X}$-дискретный модуль, то кольцо $\operatorname{End}(M)$ полурегулярно и $J(\operatorname{End}(M))=\nabla(M)$.

Доказательство. Пусть $T=\operatorname{End}(M)$ и $\nabla=\nabla(M)$. Рассмотрим произвольный эндоморфизм $\alpha: M \rightarrow M$. Тогда для некоторого эндоморфизма $\beta: X \rightarrow X$ выполнено равенство $p \beta=\alpha p$. Пусть $\bar{S}=S / J(S)$. Для элемента $y \in S$ через $\bar{y}$ будем обозначать смежный класс $y+J(S)$. Аналогичных обозначений будем придерживаться и для кольца $T / \nabla$. Так как $\bar{S}-$ регулярное кольцо, существует такой элемент $x \in S$, что $\bar{\beta}=\bar{\beta} \bar{x} \bar{\beta}$. Пусть $\bar{e}=\bar{x} \bar{\beta}$ и $\bar{f}=\bar{\beta} \bar{x}$. Тогда $\bar{e}^{2}=\bar{e}, \bar{f}^{2}=\bar{f}$ и $\bar{\beta}=\bar{\beta} \bar{e}, \bar{f} \bar{\beta} \bar{e}=\bar{\beta}$. Поскольку идемпотенты кольца $S$ поднимаются по модулю идеала $J(S)$, без ограничения общности можно считать, что $e, f \in S$ - идемпотенты. Так как

$$
(\bar{e} \bar{x} \bar{f})(\bar{f} \bar{\beta} \bar{e})=\bar{e} \bar{x}(\bar{f} \bar{\beta} \bar{e})=\bar{e} \bar{x} \bar{\beta}=\bar{e},
$$

имеем $(e x f)(f \beta e)=e+j$ для некоторого $j \in J(S)$. Таким образом, $(e x f)(f \beta e)=$ $e+e j e$. Поскольку $e+e j e \in U(e S e)$, существует такой элемент $x^{\prime} \in S$, что $\left(e x^{\prime} f\right)(f \beta e)=e$. Таким образом, $f \beta e: e(X) \rightarrow f(X)$ - мономорфизм.

$\mathrm{C}$ другой стороны, имеют место равенства

$$
(\bar{f} \bar{\beta} \bar{e})(\bar{e} \bar{x} \bar{f})=\bar{\beta}(\bar{e} \bar{x} \bar{f})=(\bar{\beta} \bar{e} \bar{x}) \bar{f}=\bar{f} .
$$

Тогда существует $j^{\prime} \in J(S)$, для которого имеет место равенство $(f \beta e)(e x f)=$ $f+j^{\prime}$. Следовательно, $(f \beta e)\left(e x^{\prime \prime} f\right)=f$ для некоторого $x^{\prime \prime} \in S$. Таким образом, $f \beta e: e(X) \rightarrow f(X)-$ изоморфизм. Так как $\hat{e} p=p e$ и $\hat{f} p=p f$, имеет место коммутативная диаграмма

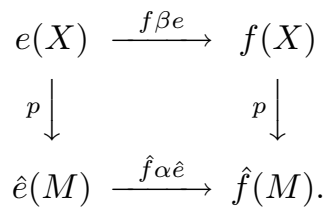

Ввиду того, что $M-\mathscr{X}$-дискретный модуль, $\hat{f} \alpha \hat{e}: \hat{e}(M) \rightarrow \hat{f}(M)-$ изоморфизм. Пусть $\hat{e} \alpha^{\prime} \hat{f}: \hat{f}(M) \rightarrow \hat{e}(M)$ - гомоморфизм, обратный к гомоморфизму $\hat{f} \alpha \hat{e}$. Тогда

$$
(\hat{f} \alpha \hat{e})\left(\hat{e} \alpha^{\prime} \hat{f}\right)=\hat{f} \quad \text { и } \quad\left(\hat{e} \alpha^{\prime} \hat{f}\right)(\hat{f} \alpha \hat{e})=\hat{e} .
$$


Пусть $\gamma=\hat{e} \alpha^{\prime} \hat{f} \in T$. Тогда

$$
\begin{aligned}
\bar{\Phi}(\overline{\alpha \gamma \alpha})=\bar{\beta} \bar{\Phi}(\bar{\gamma}) \bar{\beta}=\bar{\beta} \Phi(\gamma) \bar{f} \bar{\beta} \bar{e}=\bar{\beta} \Phi(\gamma) \Phi(\hat{f} \alpha \hat{e}) & =\bar{\beta} \Phi(\gamma(\hat{f} \alpha \hat{e}))=\bar{\beta} \Phi(\hat{e})=\bar{\beta} \bar{e}=\bar{\beta}=\bar{\Phi}(\bar{\alpha}) .
\end{aligned}
$$

Поскольку гомоморфизм $\bar{\Phi}$ инъективен, $\overline{\alpha \gamma \alpha}=\bar{\alpha}$. Таким образом, $T / \nabla-$ регулярное кольцо.

Покажем, что $J(T)=\nabla$. Так как кольцо $T / \nabla$ регулярно, $J(T) \leq \nabla$. Докажем обратное включение. Пусть $\alpha \in \nabla$ и $\beta: X \rightarrow X$ - гомоморфизм, для которого выполнено равенство $\alpha p=p \beta$. Тогда $0=\bar{\Phi}(\alpha+\nabla)=\beta+J(S)$ и, следовательно, $\beta \in J(S)$. Для произвольного $\gamma: M \rightarrow M$ пусть $\gamma^{\prime}-$ эндоморфизм модуля $X$, для которого выполнено равенство $\gamma p=p \gamma^{\prime}$. Так как $\bar{\Phi}(\alpha \gamma+\nabla)=\beta \gamma^{\prime}+J(S)=0$, то $1_{X}-\beta \gamma^{\prime}-$ изоморфизм. Поскольку модуль $M \mathscr{X}$-дискретный, $1_{M}-\alpha \gamma-$ изоморфизм для любого $\gamma \in T$. Таким образом, $\alpha \in J(T)$.

Теорема 25. Если $M-\mathscr{X}$-идемпотентно коинвариантный модуль, то модуль $M$ является $\mathscr{X}$-дискретным в точности тогда, когда $\nabla(M)=J(\operatorname{End}(M))$ и $\operatorname{End}(M) / \nabla(M)$ - регулярное кольцо.

ДокАЗАТЕЛЬСтво. Необходимость следует из теоремы 24. Предположим, что $\nabla(M)=J(\operatorname{End}(M))$ и кольцо $\operatorname{End}(M) / \nabla(M)$ регулярно. Покажем, что модуль $M \mathscr{X}$-дискретный. Пусть $T=\operatorname{End}(M)$ и $S=\operatorname{End}(X)$. Рассмотрим коммутативную диаграмму

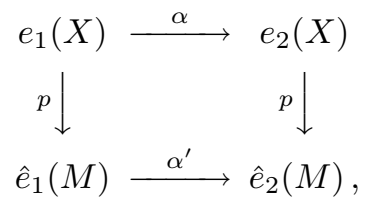

где $\alpha-$ изоморфизм и $e_{1}, e_{2}-$ идемпотенты из кольца $S$. Покажем, что $\alpha^{\prime}-$ изоморфизм. Так как $\alpha$ является изоморфизмом, для некоторого гомоморфизма $\alpha^{-1}: e_{2}(X) \rightarrow e_{1}(X)$ имеют место равенства $\alpha \alpha^{-1}=1_{e_{2}(X)}, \alpha^{-1} \alpha=1_{e_{1}(X)}$. Пусть $\gamma \in S-$ эндоморфизм, действующий по правилу $\gamma\left(e_{1} m+\left(1-e_{1}\right) m\right)=$ $\alpha e_{1} m$, и $\gamma^{\prime} \in S-$ эндоморфизм, действующий по правилу $\gamma\left(e_{2} m+\left(1-e_{2}\right) m\right)=$ $\alpha^{-1} e_{2} m$, где $m \in P$. Тогда $\gamma \gamma^{\prime}=e_{2}$ и $\gamma^{\prime} \gamma=e_{1}$. Рассмотрим эндоморфизм $\omega \in T$, действующий согласно правилу $\omega\left(\hat{e}_{1} m+\left(1-\hat{e}_{1}\right) m\right)=\alpha^{\prime} \hat{e}_{1} m$, где $m \in M$. Ясно, что $\omega=\omega \hat{e}_{1}=\hat{e}_{2} \omega$. Тогда для каждого $m \in P$ имеем

$$
p \gamma(m)=p \alpha\left(e_{1}(m)\right)=\alpha^{\prime} p\left(e_{1}(m)\right)=\alpha^{\prime} \hat{e}_{1} p(x)=\omega p(m) .
$$

Поскольку $T / J(T)$ - регулярное кольцо, для некоторого $\beta_{1} \in T$ имеет место равенство $\omega-\omega \beta_{1} \omega \in J(T)$. Для некоторого $\beta \in S$ имеет место равенство $p \beta=\beta_{1} p$. Следовательно, $\gamma-\gamma \beta \gamma \in J(S)$.

Так как $\gamma^{\prime}(\gamma-\gamma \beta \gamma) \in J(S)$, то $e_{1}-e_{1} \beta \gamma \in J(S)$. Ввиду того, что гомоморфизм $\bar{\Phi}$ инъективен, имеем $\hat{e}_{1}-\hat{e}_{1} \beta_{1} \omega \in J(T)$. Следовательно, $\hat{e}_{1}-\hat{e}_{1} \beta_{1} \omega \hat{e}_{1} \in$ $\hat{e}_{1} J(T) \hat{e}_{1}=J\left(\hat{e}_{1} T \hat{e}_{1}\right)$. Тогда $\hat{e}_{1} \beta_{1} \omega \hat{e}_{1} \in U\left(\hat{e}_{1} T \hat{e}_{1}\right)$ и существует элемент $t \in T$, для которого выполнено равенство $e_{1}^{\prime} t \omega \hat{e}_{1}=\hat{e}_{1}$. Таким образом, $\alpha^{\prime}: \hat{e}_{1}(M) \rightarrow$ $\hat{e}_{2}(M)-$ мономорфизм.

Так как $(\gamma-\gamma \beta \gamma) \gamma^{\prime} \in J(S)$, то $e_{2}-\gamma \beta e_{2} \in J(S)$. Тогда $\hat{e}_{2} \omega \beta_{1} \hat{e}_{2} \in U\left(\hat{e}_{2} T \hat{e}_{2}\right)$. Следовательно, для некоторого элемента $t^{\prime} \in T$ выполнено равенство $\hat{e}_{2} \omega t^{\prime} \hat{e}_{2}=$ $\hat{e}_{2}$. Таким образом, $\alpha^{\prime}-$ изоморфизм. 
Следствие 26. $У$ каждого неразложимого $\mathscr{X}$-дискретного модуля кольцо эндоморфизмов локально.

ДоКАЗАТЕЛЬСтво непосредственно следует из теоремы 24.

Теорема 27. Если $M-\mathscr{X}$-дискретный модуль, то $M$ является конечно заменяемым модулем.

ДоКАЗАТЕЛЬСтво непосредственно следует из теоремы 24 и $[29$, предложение 1.6].

Кольцо $R$ называется чистым, если каждый элемент $r$ из $R$ представим в виде $r=e+u$, где $e^{2}=e \in R$ и $u$ - обратимый элемент из $R$. Модуль $M$ называется чистым, если $\operatorname{End}(M)$ - чистое кольцо.

Теорема 28. Если $M-\mathscr{X}$-дискретный модуль и $\operatorname{End}(X)-$ чистое кольцо, то кольцо $\operatorname{End}(M)$ чистое.

ДокАЗАтЕльСтво. Пусть $\alpha-$ произвольный элемент из $\operatorname{End}(M)$. Существует такой эндоморфизм $\beta \in X$, что $p \beta=\alpha p$. Так как $\operatorname{End}(X)-$ чистое кольцо, для некоторого автоморфизма $\gamma$ модуля $X$ и идемпотента $e \in \operatorname{End}(X)$ выполнено равенство $\beta=e+\gamma$. Поскольку модуль $M \quad \mathscr{X}$-идемпотентно коинвариантный, $p e=e_{1} p$ для некоторого идемпотента $e_{1} \in \operatorname{End}(M)$. Пусть $\gamma^{\prime}=\alpha-e_{1} \in \operatorname{End}(M)$. Тогда $p \gamma=p(\beta-e)=p \beta-p e=\alpha p-e_{1} p=\gamma^{\prime} p$. Так как модуль $M \mathscr{X}$-дискретный, $\gamma^{\prime}$ - автоморфизм модуля $M$. Таким образом, $M$ является чистым модулем.

Модуль $M$ называется квазинепреръвным, если он инвариантен относительно идемпотентных эндоморфизмов своей инъективной оболочки. Квазинепрерывный модуль $M$ называется непрерывным, если его каждый подмодуль, изоморфный прямому слагаемому $M$, является прямым слагаемым $M$. Следующее утверждение доказывается с помощью стандартных рассуждений.

Лемма 29. Пусть $M-$ квазинепрерывный модуль и $u: M \rightarrow E(M)-$ инъективная оболочка модуля $M$. Следующие условия равносильны:

(1) $M$ - непрерывный модуль;

(2) если для идемпотентов $e_{1}, e_{2} \in \operatorname{End}(E), e_{1}^{\prime}, e_{2}^{\prime} \in \operatorname{End}(M)$ выполнены равенства $e_{i} u=u e_{i}^{\prime}, i=1,2$, для гомоморфизмов $\alpha, \alpha^{\prime}$ коммутативна диаграмма

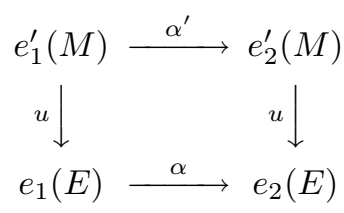

и $\alpha-$ изоморфизм, то $\alpha^{\prime}-$ изоморфизм.

Пусть $u: M \rightarrow X-\mathscr{X}$-оболочка модуля $M$. Модуль $M$ называется $\mathscr{X}$ непрерывным, если выполнены следующие условия:

(1) $M-\mathscr{X}$-идемпотентно инвариантный модуль;

(2) если для идемпотентов $e_{1}, e_{2} \in \operatorname{End}(E), e_{1}^{\prime}, e_{2}^{\prime} \in \operatorname{End}(M)$ выполнены равенства $e_{i} u=u e_{i}^{\prime}, i=1,2$, для гомоморфизмов $\alpha, \alpha^{\prime}$ коммутативна диаграмма




и $\alpha$ - изоморфизм, то $\alpha^{\prime}$ - изоморфизм.

Далее будем предполагать, что $u: M \rightarrow X-$ мономорфная $\mathscr{X}$-оболочка правого $R$-модуля $M$ и $\operatorname{End}_{R}(X)$ - полурегулярное кольцо. Имеет место кольцевой гомоморфизм $\Phi: \operatorname{End}(M) \rightarrow S / J(S)$, определенный согласно правилу $\Phi(f)=\bar{f}+J(S)$, где $\bar{f}: X \rightarrow X-$ гомоморфизм, для которого выполнено равенство $\bar{f} u=u f$. Пусть $\Delta(M)=\operatorname{Ker}(\Phi)$. Тогда имеем мономорфизм $\bar{\Phi}: M / \Delta(M) \rightarrow S / J(S)$. Несложно заметить, что если $\mathscr{X}-$ класс инъективных правых $R$-модулей, то $\Delta(M)=\left\{f \in \operatorname{End}(M) \mid \operatorname{Ker}(f) \leq_{e} M\right\}$.

Следующие утверждения являются двойственными аналогами соответственно теорем 24, 25, 27 и 28.

Теорема 30. Если $M-\mathscr{X}$-непрерывный модуль, то кольцо $\operatorname{End}(M)$ полурегулярно и $J(\operatorname{End}(M))=\Delta(M)$.

Теорема 31. Если $M-\mathscr{X}$-идемпотентно инвариантный модуль, то модуль $M \mathscr{X}$-непрерывен в точности тогда, когда $\Delta(M)=J(\operatorname{End}(M))$ и кольцо $\operatorname{End}(M) / \Delta(M)$ регулярно.

Теорема 32. Если $M-\mathscr{X}$-непрерывный модуль, то модуль $M$ конечно заменяем.

Теорема 33. Если $M-\mathscr{X}$-непрерывный модуль, то кольцо $\operatorname{End}(M)$ чистое.

\section{4. Некоторые приложения}

В качестве приложений полученных выше результатов рассмотрим случаи плоских накрытий, инъективных и чисто инъективных оболочек.

Пусть $R$ - кольцо и $\mathscr{F}-$ класс плоских правых $R$-модулей. Согласно $[30$, теорема 3] каждый правый $R$-модуль обладает плоской оболочкой. Если $R$ - совершенное справа кольцо, то согласно [25, предложение 1.3.1] каждая плоская оболочка произвольного правого $R$-модуля $M$ совпадает с проективной оболочкой $M$. Таким образом, над совершенным справа кольцом $R$ правый $R$-модуль $M$ дискретный в точности тогда, когда $M-\mathscr{F}$-дискретный модуль

Теорема 34. Пусть $M-\mathscr{F}$-дискретный правый $R$-модуль. Имеют место следующие утверждения:

(1) End $M-$ полурегулярное кольцо;

(2) $M-$ конечно заменяемый модуль;

(3) End $M$ - чистое кольцо;

(4) если $M$ - неразложимый модуль, то End $M$ - локальное кольцо.

ДокАЗАТЕльство следует из [21, лемма 5.1], теорем 24, 20 и следствия 21.

Следствие 35. Пусть $R$ - совершенное справа кольцо и $M$ - дискретный правый $R$-модуль. Имеют место следующие утверждения:

(1) End $M-$ полурегулярное кольцо и

$$
J(\operatorname{End}(M))=\{f \in \operatorname{End}(M) \mid f(M) \ll M\}
$$

(2) $M-$ конечно заменяемый модуль;

(3) End $M$ - чистое кольцо;

(4) если $M$ - неразложимый модуль, то End $M$ - локальное кольцо. 
Следствие 36. Пусть $R$ - полусовершенное справа кольцо и $M-$ конечно порожденный дискретный правый $R$-модуль. Имеют место следующие утверждения:

(1) End $M-$ полурегулярное кольцо и

$$
J(\operatorname{End}(M))=\{f \in \operatorname{End}(M) \mid f(M) \ll M\}
$$

(2) $M-$ конечно заменяемый модуль;

(3) End $M$ - чистое кольцо;

(4) если $M-$ неразложимый модуль, то End $M$ - локальное кольцо.

Теорема 37. Пусть $M-$ непрерывный правый $R$-модуль. Имеют место следующие утверждения:

(1) End $M$ - полурегулярное кольцо и

$$
J(\operatorname{End}(M))=\left\{f \in \operatorname{End}(M) \mid \operatorname{Ker}(f) \leq_{e} M\right\}
$$

(2) $M-$ конечно заменяемый модуль;

(3) End $M$ - чистое кольцо;

(4) если $M$ - неразложимый модуль, то End $M$ - локальное кольцо.

ДокАЗАТЕЛЬство следует из теорем 31, 33 и 34, если в этих теоремах положить $\mathscr{X}$ равным классу всех инъективных правых $R$-модулей.

Модуль $M$ назовем чисто непрерывным, если $M-\mathscr{X}$-непрерывный модуль, где $\mathscr{X}$ - класс чисто инъективных правых $R$-модулей. Согласно [31, предложение 6] каждый модуль обладает мономорфной чисто инъективной оболочкой, и из [32, теорема 9] следует, что кольцо эндоморфизмов каждого чисто инъективного правого модуля полурегулярное и самоинъективное справа. Тогда из теорем 24, 27, 28 и следствия 26 вытекает утверждение, которое обобщает результаты из [32] о кольцах эндоморфизмов чисто инъективных модулей.

Теорема 38. Пусть $M-$ чисто непрерывный правый $R$-модуль. Имеют место следующие утверждения:

(1) End $M-$ полурегулярное кольцо;

(2) $M-$ конечно заменяемый модуль;

(3) End $M$ - чистое кольцо;

(4) если $M-$ неразложимый модуль, то End $M$ - локальное кольцо.

ЗАмечАниЕ. Известно, что проблема Шрёдера - Бернштейна решается положительно для дискретных модулей над совершенными кольцам и непрерывных модулей [16, теорема 3.17]. Поэтому представляется интересным изучить эту проблему в общем случае для $\mathscr{X}$-непрерывных и $\mathscr{X}$-дискретных модулей. Плоские, инъективные, дискретные и непрерывные модули играют существенную роль в гомологической классификации колец. Интересно выяснить строение колец, над которыми каждый модуль $\mathscr{F}$-дискретен. Очевидно, что примерами таких колец являются регулярные кольца. Согласно [26, 40.4] над прюферовым кольцом модуль $M$ плоский в точности тогда, когда $M-$ модуль без кручения. Хорошо известны описания чисто инъективных $\mathbb{Z}$-модулей $[33$, теорема 3.2] и непрерывных $\mathbb{Z}$-модулей [16, с. 19]. Поэтому естественной является задача об описании $\mathscr{F}$-дискретных модулей и чисто непрерывных модулей над прюферовыми кольцами, в частности, над кольцом целых чисел. 


\section{ЛИТЕРАТУРА}

1. Johnson R. E., Wong E. T. Quasi-injective modules and irreducible rings // J. London Math. Soc. 1961. V. 36, N 1. P. 260-268.

2. Dickson S. E., Fuller K. R. Algebras for which every indecomposable right module is invariant in its injective envelope // Pacific J. Math. 1969. V. 31, N 3. P. 655-658.

3. Jain $S$. K., Singh $S$. On pseudo-injective modules and self pseudo injective rings // J. Math. Sci. 1967. V. 2. P. 23-31.

4. Er N., Singh S., Srivastava A. Rings and modules which are stable under automorphisms of their injective hulls // J. Algebra. 2013. V. 379. P. 223-229.

5. Абызов А. Н., Куинь Ч. К., Тай Д. Д. Дуально автоморфизм-инвариантные модули над совершенными кольцами // Сиб. мат. журн. 2017. Т. 58, № 5. С. 959-971.

6. Guil Asensio P. A., Keskin D. T., Kalebogaz B., Srivastava A. K. Modules which are coinvariant under automorphisms of their projective covers // J. Algebra. 2016. V. 466, N 15. P. 147-152.

7. Singh S., Srivastava A. K. Dual automorphism-invariant modules // J. Algebra. 2012. V. 371, N 1. P. 262-275.

8. Jeremy L. Sur les modules et anneaux quasi-continus // C. R. Acad. Sci. Paris. 1971. V. 273. P. 80-83.

9. Jeremy L. Modules et anneaux quasi-continus // Canad. Math. Bull. 1974. V. 17, N 2. P. 217-228.

10. Mohamed S., Bouhy T. Continuous modules // Arabian J. Sci. Eng. 1977. V. 2. P. 107-122.

11. Takeuchi T. On direct modules // Hokkaido Math. J. 1972. V. 1. P. 168-177.

12. Utumi Y. On continuous rings and self-injective rings // Trans. Amer. Math. Soc. 1965. V. 118. P. $158-173$.

13. Goel V. K., Jain S. K. $\pi$-Injective modules and rings whose cyclics are $\pi$-injective // Commun. Algebra. 1978. V. 6, N 1. P. 59-72.

14. Dung N. V., Huynh D. V., Smith P. F., Wisbauer R. Extending modules. Harlow: Longman Sci. Technical, 1994. (Pitman Res. Notes Math.; V. 313).

15. Clark J., Lomp C., Vanaja N., Wisbauer R. Lifting modules. Supplements and projectivity in module theory. Basel; Boston; Berlin: Birkhäuser, 2006.

16. Mohammed S. H., Müller B. J. Continuous and discrete modules. New York; Sydney: Camb. Univ. Press, 1990. (London Math. Soc. Lecture Note Ser.; V. 147).

17. Nicholson W. K., Yousif M. F. Quasi-Frobenius rings. Cambridge: Camb. Univ. Press, 2003.

18. Туганбаев A. А. Теория колец. Арифметические модули и кольца. М.: МЦНМО, 2009.

19. Birkenmeier G. F., Park J. K., Rizvi S. T. Extensions of rings and modules. New York: Springer-Verl., 2013.

20. Guil Asensio P. A., Kalebogaz B., Srivastava A. K. The Schröder-Bernstein problem for modules // J. Algebra. 2018. V. 498. P. 153-164.

21. Guil Asensio P. A., Keskin Tütüncü D., Srivastava A. K. Modules invariant under automorphisms of their covers and envelopes // Israel J. Math. 2015. V. 206, N 1. P. 457-482.

22. Guil Asensio P., Srivastava A. K., Quynh T. C. Additive unit structure of endomorphism rings and invariance of modules // Bull. Math. Sci. 2017. V. 7, N 2. P. 229-246.

23. Thuyet L. V., Dan P., Quynh T. C. Modules which are invariant under idempotents of their envelopes // Colloq. Math. 2016. V. 143, N 2. P. 237-250.

24. Enochs E. E., Jenda O. M. G. Relative homological algebra. Berlin: Walter de Gruyter, 2011. (Gruyter Expo. Math.; V. 30).

25. Xu J. Flat covers of modules. Berlin: Springer-Verl., 1996. (Lecture Notes Math.; V. 1634).

26. Wisbauer R. Foundations of module and ring theory. Philadelphia: Gordon and Breach, 1991.

27. Wu L. E., Jans J. P. On quasi projectives // Illinois J. Math. 1967. V. 11, N 2. P. 439-448.

28. Nguyen $X$. H., Zhou $Y$. Rings whose cyclic modules are lifting and $\oplus$-supplemented // Commun. Algebra. 2018. V. 46, N 11. P. 4918-4927.

29. Nicholson W. K. Lifting idempotents and exchange rings // Trans. Amer. Math. Soc. 1977. V. 229. P. 269-278.

30. Bican L., El Bashir R., Enochs E. All modules have flat covers // Bull. London Math. Soc. 2001. V. 33, N 4. P. 385-390.

31. Warfield R. B. Purity and algebraic compactness for modules // Pacif. J. Math. 1969. V. 28, N 3. P. 699-719.

32. Zimmermann-Huisgen B., Zimmermann $W$. Algebraically compact rings and modules // Math. Z. 1978. Bd 161, Heft 1. S. 81-93. 
33. Fuchs L. Abelian groups. Berlin: Springer-Verl., 2015.

Поступила в редакцию 6 ноября 2018 г.

После доработки 6 ноября 2018 г.

Принята к публикачии 12 марта 2019 г.

Абызов Адель Наилевич

Казанский (Приволжский) федеральный университет, кафедра алгебры и математической логики,

ул. Кремлевская, 18, Казань 42000

Adel.Abyzov@kpfu.ru

Le Van Thuyet (Ле Ван Тхуиет)

Department of Mathematics, College of Education,

Hue University, 34 Le Loi, Hue city, Vietnam

lvthuyet@hueuni.edu.vn

Truong Cong Quynh (Чюонг Конг Куинь)

Department of Mathematics, The University of Danang,

University of Science and Education,

459 Ton Duc Thang, Danang city, Vietnam

tcquynh@ued.udn.vn

Туганбаев Аскар Аканович

Национальный исследовательский университет «МЭИ»,

кафедра высшей математики,

ул. Красноказарменная, 14, Москва 111250

tuganbaev@gmail.com 